\title{
Desempenho de escolares com dislexia: Programas de intervenção metalinguístico e de leitura
}

\author{
Performance of students with dyslexia in \\ intervention programs: Metalinguistics and reading
}

Giseli Donadon Germano ${ }^{[a]}$, Fábio Henrique Pinheiro ${ }^{[b]}$, Simone Aparecida Capellini[c]

\footnotetext{
${ }^{[a]}$ Fonoaudióloga, doutora em Educação pelo Programa de Pós-Graduação em Educação da Faculdade de Filosofia e Ciências da Universidade Estadual Paulista Júlio de Mesquita Filho (FFC/Unesp), Marília, SP - Brasil, e-mail: giseliger@yahoo.com.br

${ }^{[b]}$ Fonoaudiólogo, doutorando do Programa de Pós-Graduação em Educação da Faculdade de Filosofia e Ciências da Universidade Estadual Paulista Júlio de Mesquita Filho (FFC/ Unesp), Marília, SP - Brasil, e-mail: fabiohenriquepinheiro@yahoo. com.br

${ }^{[c]}$ Fonoaudióloga, docente do Programa de Pós-Graduação em Educação da Faculdade de Filosofia e Ciências da Universidade Estadual Paulista Júlio de Mesquita Filho (FFC/ Unesp), Marília, SP - Brasil, e-mail: sacap@uol.com.br
}

Recebido: 28/07/2010 Received: 07/28/2010

Aprovado: 03/11/2010 Approved: 11/03/2010

\section{Resumo}

A dislexia é um distúrbio específico de aprendizagem de origem neurológica, resultante de um deficit no componente fonológico da linguagem. Este estudo visou verificar o desempenho de escolares com dislexia em programa de intervenção fonológica, programa de leitura e programa de intervenção fonológica e leitura em escolares com dislexia. Participaram deste estudo 60 disléxicos distribuídos em: GI (dez escolares com dislexia submetidos e dez escolares com dislexia não submetidos ao programa de intervenção fonológica), GII (dez escolares com dislexia submetidos e dez escolares com dislexia não submetidos ao programa de leitura), GIII (dez escolares com dislexia submetidos e dez escolares com dislexia não submetidos ao programa de intervenção fonológica e leitura). Foi realizado o programa de intervenção fonológica e leitura em três etapas: pré-testagem, treino, pós-testagem. Os resultados revelaram diferenças estatisticamente significantes entre os dois momentos de avaliação, revelando melhora nas habilidades cognitivo-linguísticas em situação de pós-testagem comparando-se a pré-testagem, demonstrando a eficácia dos três programas para escolares com dislexia. Concluiu-se que houve uma melhora do desempenho dos escolares com dislexia submetidos aos programas de intervenção, evidenciando a necessidade da instrução fonológica no contexto da alfabetização, pois isso auxiliaria os escolares a desenvolverem habilidades cognitivo-linguísticas para a aprendizagem da base alfabética do sistema de escrita do português brasileiro.

Palavras-chave: Estudos de intervenção. Dislexia. Aprendizagem. Leitura.

\begin{abstract}
Dyslexia is specific learning disabilities, of neurological origin, resulted from a phonological deficit of language. This study aims to verify the performance of students with dyslexia in phonological intervention program, reading program, and phonological and reading remediation program in students with dyslexia. The participants of this study were 60 students who were divided as follows: GI (ten students with dyslexia submitted to phonological remediation program and ten students with dyslexia not submitted to phonological remediation program), GII (ten students with dyslexia submitted to reading program and ten students with dyslexia not submitted to reading program), GIII (ten students with dyslexia submitted to phonological and reading program and ten students with dyslexia not submitted to phonological and reading program). The phonological and reading remediation was applied in three phases: pretest, training, post-testing. The results showed significant statistical difference between two evaluation moments, revealed better performance in the cognitive-linguistic
\end{abstract}


skills in post-test situation comparing to the pretest, showing the efficacy of the three remediation programs for students with dyslexia. The better performance of the students with dyslexia submitted to the remediation programs shows the necessity of phonological instruction or phonological instruction with reading to be offered in the literacy context, once this will help students to develop cognitive-linguistic skills to learn the alphabetic basis of the Brazilian Portuguese writing system.

Keywords: Intervention studies. Dyslexia. Learning. Reading.

\section{Introdução}

A dislexia é um distúrbio específico de aprendizagem de origem neurológica, caracterizada por dificuldade com a fluência correta na leitura e dificuldade na habilidade de decodificação e soletração, resultantes de um deficit no componente fonológico da linguagem (Lyon, Shaywitz \& Shaywitz, 2003).

Para que ocorra a aprendizagem da leitura escrita, faz-se necessário o desenvolvimento da consciência fonológica, correlacionada ao reconhecimento do alfabeto. 0 sistema de escrita do português caracteriza-se pela transparência ortográfica, ou seja, pela regularidade, sendo cada fonema correspondente a um e somente um grafema e vice-versa e, pela opacidade ortográfica, ou seja, pela irregularidade, com grafemas que correspondem a mais de um fonema e com fonemas que correspondem a vários grafemas (Meireles \& Correa, 2005; Mousinho \& Correa, 2009).

Embora a aquisição de leitura dependa de um amplo conjunto de habilidades de linguagem e, como outros aspectos do desenvolvimento, essa aquisição deve ser considerada em um contexto de causação múltipla, as evidências empíricas apontam que habilidades de consciência fonológica se encontram entre seus mais importantes determinantes (Bernardino, Freitas, Souza, Maranhes \& Bandini, 2006; Vaessen \& Blomert, 2010; Vloedgraven \& Verhoeven, 2009).

Escolares com dificuldade em leitura e escrita apresentam dificuldades em realizar a segmentação de palavras em partes menores (sílabas) e, por isso, não conseguem realizar a correspondência entre letra e som (soletração), e/ou não conseguem generalizar sobre a decodificação de novas palavras (Morris et al., 2010; Piasta \& Wagner, 2010).

0 escolar com dislexia pode falhar na aquisição de níveis satisfatórios de habilidades de leitura em razão dos deficit em qualquer um dos componentes desses três domínios, incluindo-se os deficit de ordem fonológica (Naples, Chang, Katz \& Grigorenko, 2009).
As habilidades fonológicas, tais como percepção de sons, identificação de rimas, combinação de sílabas e segmentação de palavras, constituem a base para leitura e escrita. Evidências indicam que quanto mais sensível a criança for aos componentes dos sons (sílabas, rimas, fonemas), melhor leitor ela será, independentemente de inteligência, recepção de vocabulário, habilidades de memória e classe social (Otaiba, Puranik, Ziolkowski \& Montgomery, 2009).

Mesmo com o passar do tempo, escolares com dislexia continuam a apresentar deficit fonológico, tornando sua leitura menos automática (exatidão de leitura), mais lentificada e requerendo muito esforço (Shaywitz \& Shaywitz, 2005).

A leitura lentificada de um texto indica pobre aquisição dessas habilidades (acesso fonológico à estrutura das palavras) e do uso da memória de trabalho, o que torna a compreensão do texto prejudicada, pois a atenção do leitor é voltada apenas para a decodificação, restando pouco recurso para o processamento das informações do texto (Miller \& Keenan, 2007).

A intervenção precoce baseada em aspectos da consciência fonológica associada à leitura de textos é considerada amplamente benéfica em escolares com baixo desempenho em leitura e escrita, pois melhora a associação entre grafemas e fonemas ou entre grafemas e maiores segmentos morfológicos (ex.: sílabas, morfemas, rimas), tornando mais eficiente o acesso e a construção de representações ortográficas (Germano \& Capellini, 2008; Kipp \& Mohr, 2008; Odegard, Ring, Smith, Biggan \& Black, 2008; O'Shaughnessy \& Swanson, 2000; Silva \& Capellini, 2010; Torgesen, Rashotte, Herron \& Lindamood, 2010; Wagner, Torgensen \& Rashotte, 1994).

No entanto, no Brasil ainda são restritos os estudos relativos aos programas de intervenção com leitura e escrita que enfoquem o desenvolvimento de habilidades cognitivo-linguísticas necessárias para a aprendizagem da leitura e da escrita e que possam ser utilizadas na prática clínica ou de pesquisa. Por isso, este 
estudo visou verificar a eficácia terapêutica do programa de intervenção fonológica, do programa de leitura e do programa de intervenção fonológica e leitura em escolares com dislexia e sua influência no desenvolvimento das habilidades cognitivo-linguísticas.

\section{Método}

Esta pesquisa foi aprovada pelo Comitê de Ética em Pesquisa da Faculdade de Filosofia e Ciências da Universidade Estadual Paulista - CEP/FFC/Unesp, sob o protocolo n. 3325/2006.

Participaram 60 escolares de $2^{\underline{a}}$ a $4^{\text {a }}$ série do Ensino Público Fundamental, sendo 80\% do gênero masculino e $20 \%$ do gênero feminino, com média etária de 10 anos e 3 meses.

- Grupo I (GI): composto por 20 escolares com o diagnóstico de dislexia, subdivididos em Grupo Ie (GIe), composto por dez escolares com dislexia submetidos ao programa de intervenção fonológica, e Grupo Ic (GIc), composto por dez escolares com dislexia não submetidos ao programa;

- Grupo II (GII): composto por 20 escolares com o diagnóstico de dislexia, subdivididos em Grupo IIe (GIIe), composto por dez escolares com dislexia submetidos ao programa de intervenção com leitura, e Grupo IIc (GIIc), composto por dez escolares com dislexia não submetidos ao programa;

- Grupo III (GIII): composto por 20 escolares com o diagnóstico de dislexia, subdivididos em Grupo IIIe (GIIIe), composto por dez escolares com bom desempenho escolar submetidos ao programa de intervenção fonológica e leitura, e Grupo IIIc (GIIIc), composto por dez escolares com dislexia que não foram submetidos ao programa.

Fizeram parte deste estudo escolares submetidos à avaliação interdisciplinar realizada por equipe de profissionais do Centro de Estudos da Educação e Saúde (CEES/Unesp-Marília) e Ambulatório de Neurologia Infantil - Aprendizagem (Hospital das Clínicas da Faculdade de Medicina da Unesp-Botucatu), incluindo avaliação fonoaudiológica, neurológica, pedagógica, neuropsicológica e de imagem (Ressonância Magnética e SPECT) e somente foram considerados disléxicos aqueles que apresentaram manifestações segundo os critérios descritos na literatura (Capellini, Padula \& Ciasca, 2004; Germano \& Capellini, 2008; Silva \& Capellini, 2010).

Este estudo foi elaborado a partir da pesquisa sobre intervenção fonológica e leitura. Em situação de pré e pós-testagem, todos os escolares foram submetidos aos mesmos procedimentos para a verificação de eficácia terapêutica dos programas utilizados.

\section{Pré-testagem}

Foram utilizados os seguintes procedimentos:

a) Termo de Consentimento Pós-Informado: conforme resolução do Conselho Nacional de Saúde CNS n. 196/96, anteriormente ao início das avaliações, os pais ou responsáveis dos pacientes selecionados assinaram o Termo de Consentimento Pós-Informado para autorização da realização do estudo.

b) Protocolo de Desempenho Cognitivo-Linguístico - versão individual: foi aplicada a versão individual do protocolo (Capellini et al., 2007). Essa versão é composta pelos seguintes subtestes: leitura de palavras e pseudopalavras, consciência fonológica (rima e aliteração), processamento auditivo (discriminação de sons, repetição de números, repetição inversa de números, repetição de palavras e repetição de não palavras), processamento visual (memória visual para formas, reversão de figuras), nomeação automática rápida (figuras e dígitos), sequenciamento (dias da semana e meses do ano). Os resultados dos subtestes da versão coletiva e individual foram obtidos por pontuação, que apresenta variação entre 1 e 40 pontos para cada acerto.

c) Leitura Oral e Compreensão de Texto: foram utilizados os textos para a leitura e compreensão de acordo com a série dos escolares. A análise da leitura dos textos foi realizada a partir de contagem dos erros das palavras lidas (exatidão de leitura), tempo total de leitura e velocidade de leitura, expressa em palavras por minuto (Capellini, 2001; Ramos, 2005).

Após a realização da pré-testagem, os escolares foram distribuídos por sorteio aleatório nos três 
programas de intervenção que tiveram o total de 22 sessões com duração de 50 minutos, sendo que duas sessões voltaram-se para a realização do procedimento de pré-testagem, 18 sessões para a realização dos procedimentos de intervenção de cada programa e duas sessões para a realização dos procedimentos de pós-testagem.

\section{Programas de intervenção}

a) Programa de intervenção fonológica

1) Identificação do som e da letra: foram apresentadas em folha de papel A4 todas as letras do alfabeto e os escolares deveriam nomear as letras e identificar os sons do alfabeto.

2) Identificação de palavras dentro de uma frase: foram apresentadas auditivamente sete frases afirmativas para os escolares dividirem cada uma em palavras, marcando-as por palmas.

3) Identificação e manipulação de sílabas na palavra: foram apresentadas auditivamente duas palavras para a identificação de sílabas iguais e, logo depois, solicitou-se à criança a manipulação de segmentação silábica para a formação de novas palavras em posição inicial, medial e final. Em cada sessão, foram fornecidas seis palavras.

4) Síntese fonêmica: foram apresentadas sete palavras auditivamente separadas por sons e os escolares deveriam reconhecer as palavras.

5) Rima: foram solicitadas aos escolares palavras que terminassem com o mesmo som. Posteriormente, foi solicitada identificação de figuras que rimassem em sua nomeação.

6) Identificação e discriminação de fonemas: foi apresentado auditivamente um fonema e solicitado que os escolares dissessem uma palavra que começasse com aquele mesmo som. Depois, foram apresentadas oralmente sete palavras e perguntou-se ao escolar se na palavra apresentada havia o fonema-alvo. Os fonemas foram apresentados seguindo a ordem do desenvolvimento de fala e linguagem.

7) Segmentação de fonemas: foi oralmente apresentada aos escolares uma palavra oralmente e eles deveriam dizer todos os fonemas dela. Em cada sessão, foram fornecidas sete palavras.
8) Subtração de fonemas: foram apresentadas aos escolares seis palavras para que retirassem o fonema final; e, em seguida, mais seis palavras para que os escolares retirassem o fonema inicial.

9) Substituição de fonemas: foi oralmente apresentada aos escolares uma palavra e eles deveriam retirar o fonema inicial e substituí-lo por outro, formando, assim, uma nova palavra. Em cada sessão, foram oferecidas sete palavras.

10) Transposição de fonemas: solicitou-se que os escolares falassem as palavras em ordem inversa da palavra apresentada para formar novas palavras.

\section{b) Programa de leitura}

Esse programa foi desenvolvido a partir da coleção de livros Estrelinha (Kinoshita, Lupker \& Rastle, 2004). A escolha dessa coleção foi realizada com base nos fatores facilitadores para a aquisição da leitura, ou seja, frequência de ocorrência da palavra na escrita; regularidade na escrita e lexicalidade vocabulário apropriado para idade e escolaridade (Junqueira, 1997). Além disso, os textos apresentam macroestrutura textual, ou seja, situação, complicação, resolução e coda (Labov \& Waletsky, 1967).

Essa coleção é composta por 18 livros de histórias, divididos em três grupos de acordo com os graus de dificuldade de leitura. Estrelinha 1: composto por seis histórias com palavras constituídas de sílabas simples (vogal ou vogal + consoante) em frases curtas e repetitivas, contendo rima. Estrelinha 2: composto por seis histórias que apresentam sílabas simples e complexas em frases curtas, repetitivas, que contêm rima. Nos livros deste grau, há predomínio de períodos simples, embora ocorra eventualmente coordenação. Estrelinha 3: composto por seis histórias com sílabas simples e complexas em frases que apresentam rima. Nos livros desse grau, predominam frases em ordem direta e de períodos simples, embora algumas vezes ocorra a coordenação ou a subordinação.

A sessão foi iniciada com a leitura de um livro de grau 1.0 grau de leitura foi modificado apenas quando o escolar apresentou $94 \%$ de exatidão (um erro ou menos em cada 20 palavras) na leitura. Dessa forma, a mudança do grau da leitura de um livro foi realizada após duas sessões de leitura de livros do 
mesmo grau com índice de porcentagem de acertos na leitura de palavras de 94\% (Clay, 1985).

c) Programa de intervenção fonológica e leitura

Este programa foi realizado por meio da vinculação de estratégias do programa de intervenção fonológica e do programa de leitura.

Cada sessão iniciou-se por meio da leitura de um livro, sendo que, após a leitura dele, as etapas do programa de intervenção fonológica foram trabalhadas sequencialmente na ordem descrita anteriormente.

\section{Pós-testagem}

Na etapa de pós-testagem do programa foram reaplicadas as avaliações da pré-testagem.

Para a análise estatística, foi utilizado o Teste dos Postos Sinalizados de Wilcoxon e o Teste de Friedman, adotando o nível de significância de 5\% $(0,05)$. A análise dos dados foi realizada com a utilização do programa SPSS (Statistical Package for Social Sciences), em sua versão 13.0.

\section{Resultados}

Na Tabela 1, quando aplicado o Teste dos Postos Sinalizados de Wilcoxon para comparar o desempenho dos escolares dos grupos deste estudo nos momentos de pré e pós-testagem, verificou-se que os três grupos submetidos à intervenção apresentaram melhora em situação de pós-testagem quando comparados na pré-testagem. Em relação ao GIe, houve diferença estatisticamente significativa na maioria dos subtestes, exceto em aliteração, ditado de palavras e de não palavras. Para os escolares do GIIe, também houve diferença estatisticamente significativa na maioria dos subtestes, exceto em cópia de formas. Já entre os escolares do GIIIe, houve diferença estatisticamente significativa em todos os subtestes (alfabeto; cópia de formas; ditado de palavras e não palavras; memória; leitura de palavras; leitura de não palavras; aliteração; contagem; repetição de palavras e não palavras; nomeação de figuras e números; memória visual duas, três, quatro e cinco; discriminação de sons; memória indireta; sequências).

Na Tabela 2, observou-se que, quando aplicado o Teste dos Postos Sinalizados de Wilcoxon para comparar o desempenho dos escolares submetidos e não submetidos nos momentos de pré e pós-testagem em leitura oral e compreensão de texto, ocorreu diferença estatisticamente significante para todas as variáveis para os GIe, GIIe e GIIIe. Entre os escolares dos grupos controles, verificou-se que não ocorreu diferença estatisticamente significante entre os escolares do GIc e GIIc para a compreensão da leitura. Os escolares do GIIIc apresentaram diferença estatisticamente significante apenas para os erros cometidos durante a leitura e a compreensão da leitura.

\section{Discussão}

Os resultados deste estudo evidenciaram que os escolares com dislexia apresentaram dificuldade quanto à leitura de palavra e pseudopalavras, leitura rápida de palavras e pseudopalavras, discriminação de sons, nomeação automática rápida, consciência fonológica e memória verbal, corroborando a descrição da literatura (Bont, Poelmans \& Blomert, 2007; Paul, Bott, Wienbruch \& Elbert, 2006; Puolakanaho et al., 2008).

A eficácia do uso dos três programas de intervenção deste estudo foi evidenciada porque escolares a estes submetidos apresentaram diferença estatisticamente significante entre os dois momentos da avaliação. Isso demonstrou melhora em situação de pós-testagem em comparação à pré-testagem quanto ao domínio das habilidades cognitivo-linguísticas avaliadas.

De modo geral, as habilidades cognitivo-linguísticas que apresentaram desempenho superior após a realização dos programas foram: reconhecimento do alfabeto, leitura de palavras, leitura de pseudopalavras, repetição de palavras e pseudopalavras, memória para dígitos, aliteração, rima, ritmo, nomeação de figuras e números, sequência de dias da semana e meses do ano, e contagem de número em ordem inversa. Isso provavelmente ocorreu por os programas enfocarem, de forma direta, habilidades de discriminação auditiva. Segundo a literatura, essa habilidade encontra-se alterada em escolares disléxicos, acarretando comprometimento no 


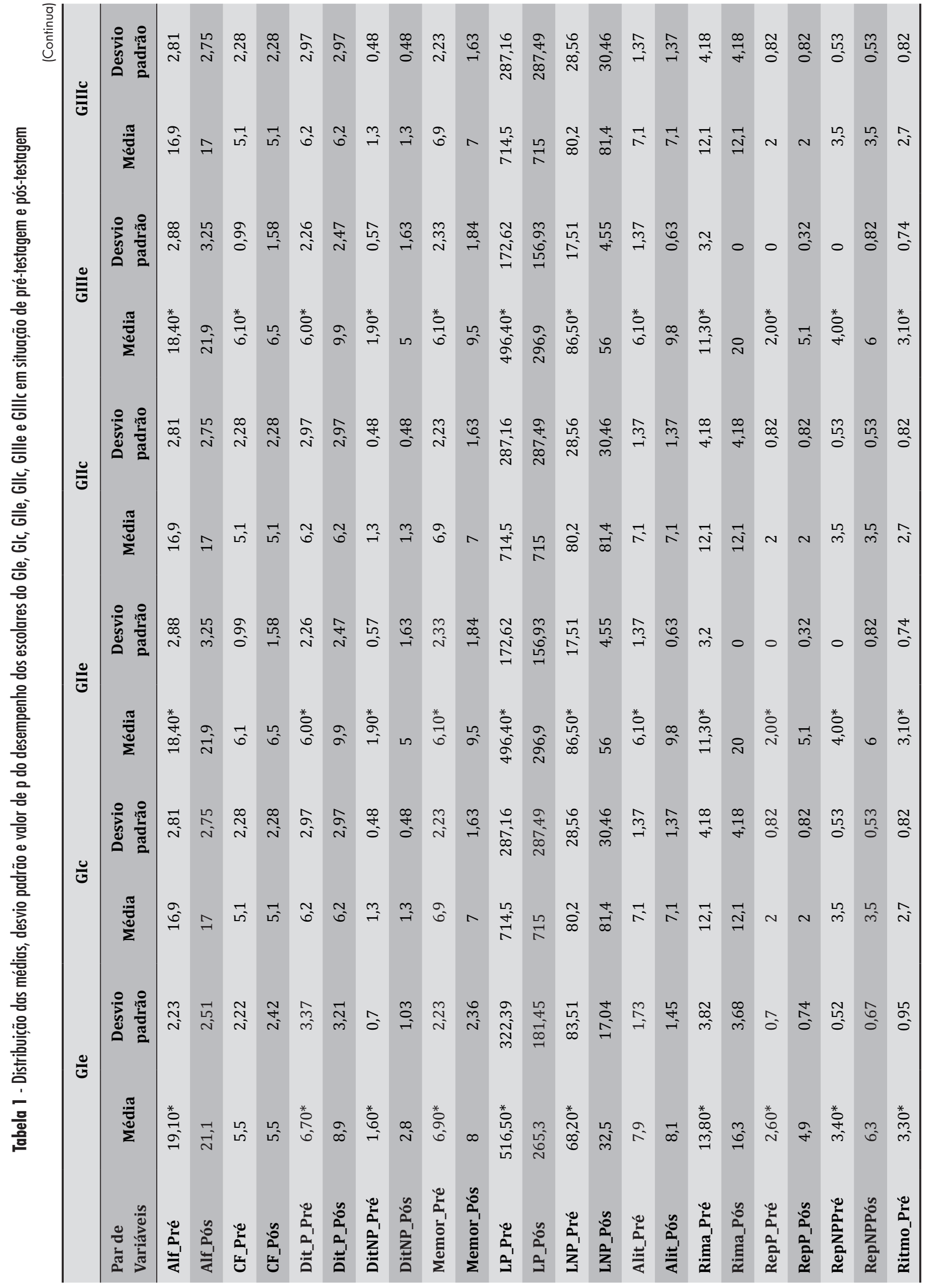




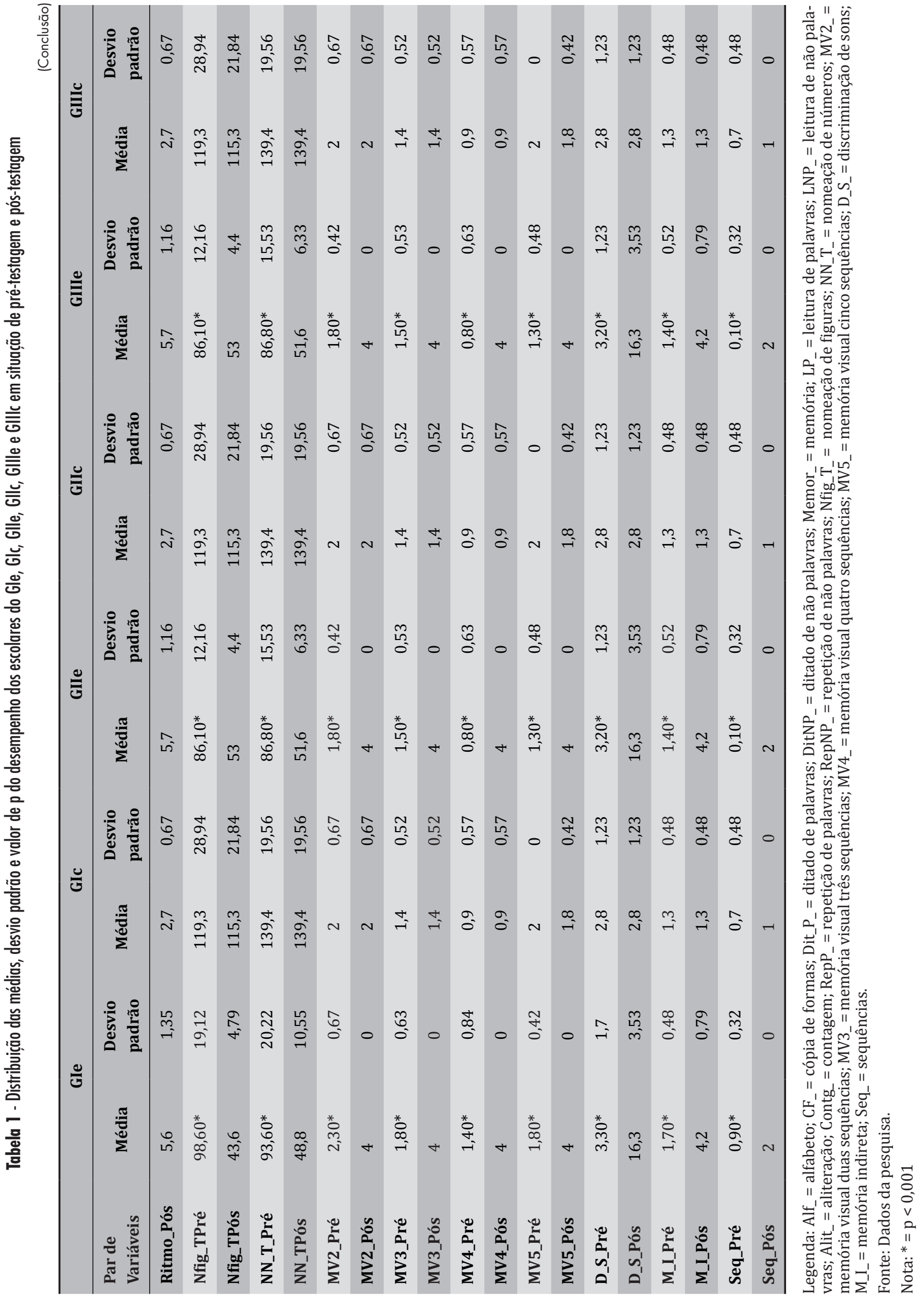


mecanismo letra-som necessário para a realização de leitura e ditado de palavras e pseudopalavras, memória e sequenciamento da informação (Boets, Wounters, Wieringen, \& Ghesquière, 2007; Dufor, Serniclaes, Sprenger-Charolles \& Démonet, 2007; Miller \& Keenan, 2007; Morris et al., 2010; Piasta \& Wagner, 2010).

A literatura indica que a instrução explícita de aspectos da consciência fonológica auxilia a descoberta do princípio alfabético. Assim, os programas de intervenção utilizados neste estudo, com enfoque fonológico, em leitura ou fonológico associado à leitura, abordaram atenção, discriminação e memória auditiva de forma direta em todas as sessões, tanto pela especificidade da tarefa quanto pela leitura em voz alta. Isso favoreceu o desenvolvimento da atenção à fonologia da língua portuguesa, conforme descrito em estudos nacionais e internacionais (Germano \& Capellini, 2008; Kipp \& Mohr, 2008; Silva \& Capellini, 2010; O'Shaughnessy \& Swanson, 2000; Odegard, Ring, Smith, Biggan \& Black, 2008; Torgensen, Wagner, Rashotte \& Lindamood, 2010; Wagner, Torgensen \& Rashotte, 1994).

Em situação de pós-testagem, pôde-se observar que todos os grupos submetidos aos programas melhoram seu desempenho em subtestes de memória de trabalho, como repetição de não palavras, nomeação automática rápida e memória para dígitos. Segundo Boets et al. (2007) e Serrano e Defior (2008), a memória fonológica não é muito eficiente nos escolares com dislexia, porém, eles podem se beneficiar com a instrução fonológica ou combinada, pois isso gera impacto direto na compreensão da leitura desses escolares.

A eficácia do programa de intervenção fonológica também foi evidenciada na leitura e na compreensão do texto lido dos escolares com dislexia submetidos aos programas. Esses escolares apresentaram desempenho superior após a realização dos programas, ocorrendo diminuição do número de palavras lidas por minuto, diminuição do tempo total de leitura e compreensão do texto lido, demonstrando, assim, que o trabalho de atenuação junto aos deficit no processamento fonológico pode resultar em diminuição das dificuldades de leitura (Naples, Chang, Katz \& Grigorenko, 2009). Entretanto, apesar de os escolares com dislexia dos grupos controles não terem sido submetidos aos programas de intervenção, apresentaram melhora na compreensão do texto lido.
Tabela 2 - Distribuição das médias, desvio padrão e valor de p do desempenho dos escolares do Gle, Glc, Glle, Gllc, Gllle, Glllc em situação de pré-testagem e pós-testagem para leitura $\mathrm{e}$ compreensão do texto lido

\begin{tabular}{|c|c|c|c|c|}
\hline & $\begin{array}{l}\text { Par de } \\
\text { Variáveis }\end{array}$ & Média & $\begin{array}{l}\text { Desvio } \\
\text { padrão }\end{array}$ & $\begin{array}{c}\text { Valor } \\
\text { de } p\end{array}$ \\
\hline \multirow{8}{*}{ GIe } & Erros_Pré & 9,9 & 4,68 & \\
\hline & Erros_Pós & 1,4 & 1,96 & $0,005^{*}$ \\
\hline & TT_Pré & 1166,89 & 470,74 & \\
\hline & TT_Pós & 636,4 & 325,46 & $0,008 *$ \\
\hline & Vel_Pré & 94,58 & 27,58 & \\
\hline & Vel_Pós & 80,56 & 23,2 & $0,005^{*}$ \\
\hline & Comp_Pré & 30 & 22,97 & \\
\hline & Comp_Pós & 52,5 & 34,26 & $0,024 *$ \\
\hline \multirow{8}{*}{ GIc } & Erros_Pré & 15 & 5,68 & \\
\hline & Erros_Pós & 14,3 & 5,79 & $0,038^{*}$ \\
\hline & TT_Pré & 482 & 38,63 & \\
\hline & TT_Pós & 458,3 & 43,61 & $0,007 *$ \\
\hline & Vel_Pré & 33,19 & 10,42 & \\
\hline & Vel_Pós & 41,92 & 14,89 & $0,011^{*}$ \\
\hline & Comp_Pré & 42,5 & 20,58 & \\
\hline & Comp_Pós & 45 & 19,72 & 0,317 \\
\hline \multirow{8}{*}{ GIIe } & Erros_Pré & 17,3 & 5,19 & \\
\hline & Erros_Pós & 3 & 2,91 & $0,005^{*}$ \\
\hline & TT_Pré & 914,3 & 406,6 & \\
\hline & TT_Pós & 498,6 & 158,89 & $0,005^{*}$ \\
\hline & Vel_Pré & 93,36 & 23,47 & \\
\hline & Vel_Pós & 72,51 & 20,72 & $0,005^{*}$ \\
\hline & Comp_Pré & 17,5 & 16,87 & \\
\hline & Comp_Pós & 57,5 & 31,29 & $0,004 *$ \\
\hline \multirow{8}{*}{ GIIc } & Erros_Pré & 15 & 5,68 & \\
\hline & Erros_Pós & 14,3 & 5,79 & $0,038 *$ \\
\hline & TT_Pré & 482 & 38,63 & \\
\hline & TT_Pós & 458,3 & 43,61 & $0,007 *$ \\
\hline & Vel_Pré & 33,19 & 10,42 & \\
\hline & Vel_Pós & 41,92 & 14,89 & $0,011^{*}$ \\
\hline & Comp_Pré & 42,5 & 20,58 & \\
\hline & Comp Pós & 45 & 19,72 & 0,317 \\
\hline
\end{tabular}


Tabela 2 - Distribuição das médias, desvio padrão e valor de p do desempenho dos escolares do Gle, Glc, Glle, Gllc, Gllle, Glllc em situação de pré-testagem e pós-testagem para leitura $\mathrm{e}$ compreensão do texto lido

\begin{tabular}{|c|c|c|c|c|}
\hline & $\begin{array}{l}\text { Par de } \\
\text { Variáveis }\end{array}$ & Média & $\begin{array}{l}\text { Desvio } \\
\text { padrão }\end{array}$ & $\begin{array}{c}\text { Valor } \\
\text { de } p\end{array}$ \\
\hline \multirow{8}{*}{ GIIIe } & Erros_Pré & 15 & 5,68 & \multirow{3}{*}{$0,038^{*}$} \\
\hline & Erros_Pós & 14,3 & 5,79 & \\
\hline & TT_Pré & 482 & 38,63 & \\
\hline & TT_Pós & 458,3 & 43,61 & $0,007 *$ \\
\hline & Vel_Pré & 33,19 & 10,42 & \multirow{3}{*}{$0,011^{*}$} \\
\hline & Vel_Pós & 41,92 & 14,89 & \\
\hline & Comp_Pré & 42,5 & 20,58 & \\
\hline & Comp_Pós & 45 & 19,72 & 0,317 \\
\hline \multirow{8}{*}{ GIIIc } & Erros_Pré & 14,2 & 3,71 & \multirow{3}{*}{$0,005^{*}$} \\
\hline & Erros_Pós & 4,3 & 2,11 & \\
\hline & TT_Pré & 169,8 & 58,12 & \\
\hline & TT_Pós & 161,1 & 43,41 & 0,284 \\
\hline & Vel_Pré & 97,87 & 32,45 & \multirow{3}{*}{0,153} \\
\hline & Vel_Pós & 100,91 & 32,31 & \\
\hline & Comp_Pré & 87,5 & 17,68 & \\
\hline & Comp Pós & 97,5 & 7,91 & $0,046^{*}$ \\
\hline
\end{tabular}

Legenda: TT = tempo total de leitura; Vel = velocidade de leitura; Comp = compreensão.

Fonte: Dados da pesquisa.

Os achados deste estudo evidenciaram que o uso de estratégias fonológicas foi eficaz para o desenvolvimento e melhora das condições de leitura, demonstrando que a instrução fonológica deve ser direcionada para a aprendizagem da leitura e, consequentemente, para a compreensão da leitura, sendo este um trabalho que, segundo a literatura internacional, apresenta benefícios quando enfatizado já na pré-escola (Joffe \& Pring, 2008; Keilmann \& Wintermeyer, 2008; Magnan \& Ecalle, 2006; Regtvoort \& Leij, 2007; Ukrainetz, Nuspl, Wilkerson \& Beddes, 2010).

Entretanto, há um aspecto deste estudo importante a ser considerado: os escolares disléxicos não submetidos aos programas também melhoraram quanto à compreensão do texto lido, comprovando que o papel da escola foi fundamental para a ocorrência desse fenômeno. Contudo, o mesmo não ocorreu com o tempo de leitura, o que demonstra falta de concordância entre o trabalho em sala de aula no que se refere à fluência e compreensão de leitura, conforme descrito na literatura (Alves, Reis, Pinheiro \& Capellini, 2009).

Os resultados deste estudo apontam para a necessidade de refletir sobre a metodologia de alfabetização utilizada em situação de sala de aula, pois, como já descrito, as habilidades de atenção, discriminação e percepção dos sons trabalhadas nos programas proporcionaram aumento na sensibilidade fonológica, o que refletiu também na melhora do tempo e compreensão da leitura.

Pesquisas informaram que a consciência fonêmica se desenvolve em virtude da alfabetização, em torno dos 6 ou 7 anos. Destaca-se que o sistema alfabético, independente da língua, não representa diretamente o significado das palavras, mas as sequências de seus sons, ou seja, uma sequência fonológica ou, mais especificamente, fonêmica. Dessa forma, tal instrução deve ocorrer em período inicial de alfabetização (Cielo, 2002; Godoy, 2003; Morais, 1998; Mota \& Melo Filha, 2009; Nicolielo, Fernandes, Garcia \& Hage, 2008; Paula, Mota \& Keske-Soares, 2005).

Essas habilidades deveriam estar inseridas no contexto de sala de aula, pois os escolares disléxicos, como os demais, poderiam ser beneficiados de um ensino voltado para a percepção fonológica do sistema de escrita do português brasileiro, melhorando o desempenho na decodificação, aumentando o vocabulário auditivo-visual e a compreensão da leitura (Banai \& Ahissar, 2006; Capellini, Germano \& Vieira-Cardoso, 2008; Piasta \& Wagner, 2010; Puolakanaho et al., 2008).

\section{Conclusão}

Os achados deste estudo permitiram concluir que:

- a realização do programa de intervenção fonológica, do programa de intervenção com leitura e do programa de intervenção fonológica e leitura foram eficazes para os escolares com dislexia deste estudo, haja vista a melhora das habilidades cognitivo-linguísticas em situação de pós-testagem em relação à pré-testagem. 
- a melhora dos escolares com dislexia submetidos aos programas de intervenção evidenciou a necessidade de a instrução fonológica ou a instrução fonológica associada à leitura ser oferecida no contexto da alfabetização, pois isso auxiliaria esses escolares a desenvolverem habilidades cognitivo-linguísticas para a aprendizagem da base alfabética do sistema de escrita do português brasileiro.

\section{Referências}

Alves, L. M., Reis, C., Pinheiro, A. M. V., \& Capellini, S. A. (2009). Aspectos prosódicos temporais da leitura de escolares com dislexia do desenvolvimento. Revista da Sociedade Brasileira de Fonoaudiologia, 14(2), 197-204. doi:10.1590/S1516-80342009000200010.

Banai, K., \& Ahissar, M. (2006). Auditory processing deficits in dyslexia: Task or stimulu related? Cerebral Cortex, 16(12), 1718-1728. doi:10.1093/cercor/ bhj107.

Bernardino, J. A., Freitas, F. R., Souza, D. G., Maranhe, E. A., \& Bandini, H. H. M. (2006). Aquisição de leitura e escrita como resultado do ensino de habilidades de consciência fonológica. Revista Brasileira de Educação Especial, 12(3), 423-450.

Boets, B., Wounters, J., Wieringen, A., \& Ghesquière, P. (2007). Auditory processing, speech perception and phonological ability in pre-school children at high-risk for dyslexia: A longitudinal study of the auditory temporal processing theory. Neuropsychologia, 45(8), 1608-1620. doi:10.1016/j. neuropsychologia.2007.01.009.

Bont, M. L., Poelmans, H., \& Blomert, L. (2007). Deviant neurophysiological responses to phonological regularities in speech in dyslexic children. Neuropsychologia. 45(7), 1427-1437. doi:10.1016/j. neuropsychologia.2006.11.009.

Capellini, S. A. (2001). Eficácia do programa de intervenção fonológica em escolares com distúrbio específico de leitura e distúrbio de aprendizagem. Tese de doutorado, Faculdade de Ciências Médicas da Universidade Estadual de Campinas, Campinas.
Capellini, S. A., Padula, N. A. M. R., \& Ciasca, S. M. (2004). Desempenho de escolares com distúrbio específico de leitura em programa de intervenção. Pró-Fono: Revista de Atualização Científica, 16(3), 261-274.

Capellini, S. A., Silva, C., Gonzaga, J., Tegeiro, M. G., Villa, P. C., \& Smythe, I. (2007). Desempenho cognitivo-linguístico de escolares de 1a a 4a séries do ensino público municipal. Revista Psicopedagogia, 24(73), 30-44.

Capellini, S. A., Germano, G. D., \& Vieira-Cardoso, A. C. (2008). Relação entre habilidades auditivas e fonológicas em crianças com dislexia do desenvolvimento. Psicologia Escolar Educacional, 12(1), 235-253. doi:10.1590/S1413-85572008000100016.

Cielo, C. A. (2002). Habilidades em consciência fonológica em crianças de 04 a 08 anos de idade. Pró-Fono: Revista de Atualização Científica, 14(3), 301-312.

Clay, M. M. (1985). The early detection of reading difficulties. Oxford: Heinemann Educational.

Dufor, O., Serniclaes, W., Sprenger-Charolles, L., \& Démonet, J. F. (2007). Top-down processes during auditory phoneme categorization in dyslexia: A PET study. Neuroimage, 46(1), 241-248. doi:10.1016/j. neuroimage.2009.01.035.

Germano, G. D., \& Capellini, S. A. (2008). Eficácia do programa de intervenção auditivo-visual computadorizado em escolares com dislexia. Pró-Fono: Revista de Atualização Científica, 20(4), 237-242. doi:10.1590/ S0104-56872008000400006.

Godoy, D. M. A. (2003). O papel da consciência fonológica no processo de alfabetização. Pró-Fono: Revista de Atualização Científica, 15(3), 241-250.

Joffe, V., \& Pring, T. (2008). Children with phonological problems: A survey of clinical practice. International Journal of Language \& Communication Disorders, 43(2), 154-164. doi:10.1080/13682820701660259.

Junqueira, S. (1997). Coleção de livros estrelinha. São Paulo: Ática.

Keilmann, A., \& Wintermeyer, M. (2008). Is a specialised training of phonological awareness indicated in every preschool child? Folia Phoniatrica et Logopaedica, 60(2), 73-79. http://dx.doi.org/10.1159/000114648 
Kinoshita, S., Lupker, S. J., \& Rastle, K. (2004). Modulation of regularity and lexicality effects in reading aloud. Memory \& Cognition, 32(8), 1255-1264. doi:10.3758/ BF03206316.

Kipp, K. H., \& Mohr, G. (2008). Remediation of developmental dyslexia: Tackling a basic memory deficit. Cognitive Neuropsychology, 25(1), 38-55. doi:10.1080/02643290701836138.

Labov, W., \& Waletsky, J. (1967). Narrative analysis: Oral versions of personal experience. In: J. Helm. Essays on verbal and visual arts. Seattle and London: University of Washington Press.

Lyon, G. R., Shaywitz, S. E., \& Shaywitz, B. A. (2003). Defining dyslexia, comorbidity, teacher's knowledge of language and reading. Annals Dyslexia, 53, 1-14. doi:10.1007/s11881-003-0001-9.

Magnan, A., \& Ecalle, J. (2006). Audio-training in children with reading disabilities. Computers and Education, 46(4), 407-425. doi:10.1016/j. compedu.2004.08.008.

Meireles, E. S., \& Correa, J. (2005). Regras contextuais e morfossintáticas na aquisição da ortografia da língua portuguesa por crianças. Psicologia: Teoria e Pesquisa, 21(1), 77-84. doi:10.1590/ S0102-37722005000100011.

Miller, A. C., \& Keenan, J. M. (2007). Phonological processing and emergent literacy in younger and older preschool children. Annals of Dyslexia, 57, 113-137. doi:10.1007/s11881-007-0008-8.

Morais, J. (1998). Alphabetic literacy and psychological structure. Letras de Hije, 33(4), 61-79.

Morris, R. D., Lovett, M. W., Wolf, M., Sevcik, R. A., Steinbach, K. A., \& Frijters, J. C., et al. Multiple-component remediation for developmental reading disabilities: IQ, socioeconomic status, and raceas factors in remedial outcome. Journal of Learning Disabilities, 46(2), 99-127.

Mota, H. B., \& Melo Filha, M. G. C. (2009). Habilidades em consciência fonológica de sujeitos após realização de terapia fonológica. Pró-Fono: Revista de Atualização Científica, 21(2), 119-124. doi:10.1590/ S0104-56872009000200006.
Mousinho, R., \& Correa, J. (2009). Conhecimento ortográfico na dislexia fonológica. In: T. Barbosa (Org.). Temas em dislexia. (pp. 33-45). São Paulo: Artes Médicas.

Naples, A. J., Chang, J. T., Katz, L., \& Grigorenko E. L. (2009). Same or different? Insights into the Etiology of Phonological Awareness and Rapid Naming. Biological Psychology, 80(2), 226-239. doi:10.1016/j. biopsycho.2008.10.002.

Nicolielo, A. P., Fernandes, G. B., Garcia, V. L., \& Hage, S. R. V. (2008). Desempenho escolar de crianças com distúrbio específico de linguagem: Relações com habilidades metafonológicas e memória de curto prazo. Revista da Sociedade Brasileira de Fonoaudiologia, 13(3), 246-250. doi:10.1590/ S1516-80342008000300008.

O’Shaughnessy, T. E., \& Swanson, H. L. (2000). A comparison of two reading interventions for children with reading disabilities. Journal of Learning Disabilities, 33(3), 257-277. doi:10.1177/00222194000330030 4.

Odegard, T. N., Ring, J., Smith, S., Biggan, J., \& Black, J. (2008). Differentiating the neural response to intervention in children with developmental dyslexia. Annals Dyslexia, 58(1), 1-14. doi:10.1007/ s11881-008-0014-5.

Otaiba, S. A., Puranik, C., Ziolkowski, R., \& Montgomery, T. (2009). Effectiveness of early phonological awareness interventions for students with speech or language impairments. The Journal of Special Education, 43(2), 107-128. doi:10.1177/0022466908314869.

Paul, I., Bott, C., Wienbruch, C., \& Elbert, T. R. (2006). Word processing differences between dyslexic and control children. BMC Psychiatry, 6(5), 1-12.

Paula, G. R., Mota, H. B., \& Keske-Soares, M. A. (2005). A terapia em consciência fonológica no processo de alfabetização. Pró-Fono: Revista de Atualização Científica, 17(2), 175-184. doi:10.1590/ S0104-56872005000200006.

Piasta, S. B., \& Wagner, R. K. (2010). Learning letter names and sounds: Effects of instruction, letter type, and phonological processing skill. Journal of experimental Child Psychology, 105(4), 324-344. doi:10.1016/j. jecp.2009.12.008. 
Puolakanaho A, Ahonen T, Aro M, Eklund K, Leppänen PH, Poikkeus A. M, et al. (2008). Developmental links of very early phonological and language skills to second grade reading outcomes: Strong to accuracy but only minor to fluency. Journal of Learning Disabilities, 41(4), 353-370. doi:10.1177/0022219407311747.

Ramos, C. S. (2005). Avaliação de leitura em escolares com indicação de dificuldade de leitura e escrita. Dissertação de mestrado, Universidade Federal de São Paulo, São Paulo.

Regtvoort, A. G. F. M., \& Leij, A. (2007). Early intervention with children of dyslexic parents: Effects of computer-based reading instruction at home on literacy acquisition. Learning and Individual Differences, 17(4), 35-53. doi:10.1016/j.lindif.2007.01.005.

Serrano, F., \& Defior, S. (2008). Dyslexia speed problems in a transparent orthography. Annals Dyslexia, 58(1), 81-95. doi:10.1007/s11881-008-0013-6.

Shaywitz, S., \& Shaywitz, B. (2005). Dyslexia (Specific reading Disability). Biological Psychiatry, 57, 301309. doi:10.1016/j.biopsych.2005.01.043.

Silva, C., \& Capellini, S. A. (2010). Eficácia do programa de remediação fonológica e leitura no distúrbio de aprendizagem. Pró-Fono: Revista de Atualização Científica, 22(2), 131-138. doi:10.1590/ S0104-56872010000200011.
Torgensen, J. K., Wagner, R. K., Rashotte, C. A., \& Lindamood, P. (2010). Computer-assisted instruction to prevent early reading difficulties in students at risk for dyslexia: Outcomes from two instructional approaches. Annals of Dyslexia, 60, 40-56. doi:10.1007/ s11881-009-0032-y.

Ukrainetz, T. A., Nuspl, J. J., Wilkerson, K., \& Beddes, S. R. (2010). The effects of syllable instruction on phonemic awareness in preschoolers. Early Childhood Research Quarterly. 26(1) 50-60. doi:10.1016/j.ecresq.2010.04.006. doi:10.1016/j. ecresq.2010.04.006.

Vaessen, A., \& Blomert, L. (2010). Long-term cognitive dynamics of fluent reading development. Journal of Experimental Child Psychology, 105(3), 213-231. doi:10.1016/j.jecp.2009.11.005.

Vloedgraven, J., \& Verhoeven, L. (2009). The nature of phonological awareness throughout the elementary grades: An item response theory perspective. Learning and Individual Differences, 19, 161-169. doi:10.1016/j.lindif.2008.09.005.

Wagner, R. K., Torgesen, J. K., \& Rashotte, C. A. (1994). Longitudinal studies of phonological processing and reading. Journal of Learning Disabilities, 27(5), 276286. doi:10.1177/002221949402700503. 